\title{
Regulatory circuits in cell motility
}

\author{
Avril Somlyo
}

Published online: 1 December 2013

(C) Springer Science+Business Media Dordrecht 2013

Regulatory Circuits in Cell Motility, a symposium celebration sponsored by the Physiological Society of Philadelphia and the Thomas Jefferson University Department of Molecular Physiology and Biophysics in honor of David Hartshorne and his many contributions to muscle research was held at Jefferson Medical College, Thomas Jefferson University in Philadelphia on October 11-12, 2013. Drs. Marion Siegman, Masumi Eto, Mike Walsh, Feri Erdodi, Tim Haystead and Avril Somlyo served on the Organizing Committee. Many colleagues and muscle researchers from Europe, Japan, Canada and the USA attended this meeting in recognition of Dr. Hartshorne's seminal contributions to the biochemistry and physiology of muscle proteins over a stellar career.

Dr. Hartshorne trained under the mentorship of the renowned Sam Perry at the University of Birmingham, England where he received his Ph.D. in Muscle Biochemistry in 1962. Following postdoctoral training at UCSF, SUNY Brooklyn and the University of Birmingham, he accepted a position as Assistant Professor of Chemistry at Carnegie-Mellon University rising through the ranks to Professor. Subsequently, he moved to Tucson at the University of Arizona where his laboratory attracted many young international muscle biochemists and physiologists. He has recently retired to enjoy the Tucson sunshine.

Apart from his early work on troponin, tropomyosin and the subunit structure of myosin, Dave is recognized for his groundbreaking contributions to the understanding of the role of myosin regulatory light chain phosphorylation as the switch to activate smooth muscle myosin actomyosin ATPase as well as the importance of calmodulin as a regulatory component of myosin light chain kinase. He is also recognized for his major studies on myosin phosphatases and their regulation by phosphorylation in smooth muscle contractility, in mitosis and cell motility. His discovery of calyculin A as an inhibitor of type 1 and 2A protein serine/threonine phosphatases has provided an excellent tool for the field.

The symposium covered the following areas: myosin light chain kinases and the roles of myosin light chain phosphorylation; regulation and function of myosin light chain phosphatase; calcium sensitization and desensitization; calmodulin, twitchin, phosphatase targeting and actin polymerization; unconventional myosins. Speaker and poster abstracts follow. 